\title{
Patient-specific assays based on whole-genome sequencing data to measure residual disease in children with acute lymphoblastic leukemia - a proof-of- concept study
}

C Arthur ( $\sim$ Cecilia.arthur@ki.se )

Karolinska Institutet

F Rezayee

Karolinska Institutet

N Mogensen

Karolinska Institutet

L Saft

Karolinska Institutet

R Rosenquist

Karolinska Institutet

M Nordenskjöld

Karolinska Institutet

A Harila-Saari

Uppsala University

E Tham

Karolinska University Hospital

G Barbany

Karolinska University Hospital

\section{Method Article}

Keywords: Liquid biopsy, whole-genome sequencing, structural variation, technical feasibility, precision medicine, diagnostic performance, acute lymphoblastic leukemia, measurable residual disease.

Posted Date: March 7th, 2022

DOI: https://doi.org/10.21203/rs.3.rs-1403708/v1

License: (9) This work is licensed under a Creative Commons Attribution 4.0 International License. Read Full License 
Version of Record: A version of this preprint was published at Frontiers in Oncology on July 5th, 2022. See the published version at https://doi.org/10.3389/fonc.2022.899325. 


\section{Abstract}

Risk-adapted treatment in acute lymphoblastic leukemia (ALL) relies on genetic information and measurable residual disease (MRD) monitoring. In this proof-of-concept study, DNA from diagnostic bone marrow from six children with ALL, without stratifying genetics or CNS involvement, underwent wholegenome sequencing to identify structural variants (SVs) in the leukemic blasts. Unique sequences generated by SVs were targeted with patient-specific droplet digital PCR assays, and genomic and cellfree DNA (cfDNA) from bone marrow, plasma and cerebrospinal fluid were analyzed longitudinally. The results indicate that this approach is a feasible option for accurate MRD quantification and that cfDNA may contribute valuable information regarding MRD and low-grade CNS involvement.

\section{Background}

The outcome of acute lymphoblastic leukemia (ALL), the most common form of childhood leukemia, has improved dramatically over the last decades. A key factor to this success has been the implementation of risk-adapted treatment intensity, based on biological and genetic factors as well as on response to induction treatment[1]. The genetic aberrations of the leukemic cells contribute important information for risk stratification of ALL patients. Consequently, modern protocols require an extensive genetic characterization of the blasts at diagnosis to correctly stratify the patients to distinct genetic risk categories. This is a labor-intensive process due to the heterogeneous nature of the genetic aberrations, requiring a range of methods for each patient.

The persistence of leukemic cells in the bone marrow (BM) after induction treatment, so called measurable residual disease (MRD), is the most important prognostic factor in $A L L[2,3]$. The detection of leukemic blasts by flow cytometry (FCM) is based on the quantification of cells with leukemia-associated immunophenotype (LAIP) while molecular techniques, such as real-time quantitative polymerase chain reaction (RQ-PCR), rely on the detection and quantification of the clonal immunoglobulin and T-cell receptor (IG/TR) gene rearrangements[4, 5]. Because of FCM's short turnover time, the use of this method is widespread but not all patients have LAIPs that allow for accurate and sensitive MRD detection. Utilizing IG/TR gene rearrangements to measure MRD has been a gold standard approach in many treatment protocols[5]. The method, however, is demanding and requires expert laboratories to achieve the desired reproducibility and sensitivity[6]. Thus, it is challenging to allocate the patients to the genetic risk categories and provide all patients with a precision marker for MRD monitoring with the currently used methods.

CNS involvement is detected in $3-10 \%$ of ALL patients at diagnosis, however the CNS may act as a sanctuary for leukemic cells and all patients are given prophylactic intrathecal treatment[7, 8]. If CNS involvement is demonstrated, treatment is intensified, hence morphologic examination of cerebrospinal fluid (CSF) is mandatory in ALL. 
High-throughput sequencing technologies have dramatically expanded our knowledge regarding the genetic events that drive leukemic transformation and whole-genome sequencing (WGS) and transcriptome profiling has successfully identified putative drivers in the majority of patients[9]. Also, WGS has shown promising potential in the diagnostic setting of hematological malignancies and detects clinically relevant genomic aberrations beyond the resolution of conventional methods[10-15].

Cell free DNA (cfDNA), and more specifically cell free tumor DNA (ctDNA), has shown promise as a biomarker for cancer in liquid biopsies yet only a handful studies have explored the potential role of plasma DNA as a marker for MRD quantification in acute leukemias. Schwartz and coworkers showed that in ALL the elevation of total cfDNA in plasma, without differentiating between ctDNA and normal cfDNA, could potentially be used as MRD marker. The same study also showed that quantitative analysis of IG/TR gene rearrangements demonstrated high concordance between plasma and PB leukocytes[16]. Another study on pediatric ALL investigated the clonal IG/TR gene rearrangements in plasma and compared the level to MRD assessed in BM by FCM. The methods showed poor correlation and yet the authors concluded that both approaches could independently predict relapse[17].

In this proof of principle study, we challenge WGS to detect genomic aberrations in pediatric ALL and use the novel sequences, created by the structural rearrangement junction sites, as leukemia-specific molecular targets. Patient-specific droplet digital polymerase chain reaction (ddPCR) assays directed towards these targets were designed and used to measure the level of disease in BM samples taken at diagnosis and follow up, as well as to investigate the presence of these targets in the cfDNA shed into the plasma and CSF before and during therapy. Finally, results were compared to MRD findings from routine methods.

\section{Methods}

\section{Patients and samples}

Four patients with precursor B-cell ALL (BCP-ALL) and two patients with T-ALL were included in the study. All were diagnosed during 2018-2019 and treated according to the NOPHO 2008 protocol [18] at Karolinska University Hospital, Stockholm, Sweden. Routine investigations of the diagnostic BM samples included morphology, FCM, chromosome banding analysis (CBA), fluorescent in situ hybridization (FISH) and comparative genome hybridization array $(\mathrm{aCGH})$. Clinical data were retrieved from medical records. None of the BCP-ALL patients had intermediate risk (IR) or high risk (HR) cytogenetic aberrations. Three of these were classified as "B-other" and one as high hyperdiploid and thus they were classified as standard risk genetics in the NOPHO 2008 ALL protocol. One patient (patient 4, B-other) suffered from two relapses. Patients 5 and 6 were included to test the approach in T-ALL. Table 1 summarizes the patients' characteristics at diagnosis.

Blood and CSF samples were collected in Cell-Free DNA BCT tubes (10 ml) (STRECK, La Vista, Nebraska) and stored at room temperature for a maximum of 5 days. Sample volumes ranged from 1-5 $\mathrm{ml}$ 
(plasma) and $0.15-4 \mathrm{ml}$ (CSF). Cell removal was performed by double centrifugation ( $10 \mathrm{~min}$ at $4^{\circ} \mathrm{C} 1,600$ $x \mathrm{G}$ and $16,000 \times \mathrm{G}$ ) and supernatants were frozen at $-80^{\circ} \mathrm{C}$. Plasma from blood donors was processed in the same manner for use as negative control. cfDNA was isolated in a semi-automated fashion using QiAamp Circulating Nucleic Acid Kit ${ }^{\text {TM }}$ on the QIAvac24 Plus vacuum manifold and the Qiacube ${ }^{\mathrm{TM}}$ (Qiagen, Manchester). cfDNA was eluted in $40 \mu \mathrm{L}$ AVE buffer and stored in $-20^{\circ} \mathrm{C}$ for a maximum of 3 months.

Diagnostic BM samples were collected in $5 \mathrm{ml}$ EDTA tubes for routine genetic investigations and followup BM samples (Ficoll separated mononuclear cells) were retrieved from the Karolinska University Hospital biobank. Genomic DNA (gDNA) was isolated using Tissue kit on EZ1 ${ }^{\text {TM }}$ Automated (Qiagen, Hilden) and stored at $-20^{\circ} \mathrm{C}$ until use. If automated gDNA extraction resulted in low yield $(<30 \mathrm{ng} / \mu \mathrm{L})$, manual isolation was performed using AllPrep DNA/RNA Mini Kit ${ }^{T M}$ (Qiagen, Hilden). gDNA negative control was extracted from peripheral blood from healthy blood donors, using QIAsymphony DSP DNA Midi Kit (Qiagen, Hilden) and anonymized and pooled prior to use.

\section{FCM and PCR based MRD analysis}

Immunophenotyping of diagnostic and follow-up BM aspirates was performed on FACSCanto/FACS Lyric flow cytometers according to the NOPHO 2008 (until September 2019) and the ALLTogether protocols [19] using standardized MRD 6- and 8-color antibody panels (Table 1 supplementary material) (all from Becton Dickinson) and the Infinicyt software program for MRD analysis. Guidelines for sample preparation and data analysis followed the NOPHO and ALLTogether laboratory guidelines. Events with LAIP were identified in all plots of interest. The final MRD value was calculated as the mean percentage of leukemic cells of all living events excluding erythropoiesis. To be considered as MRD, a cluster of cells ( $\geq 10$ events) with LAIP had to be identified. A cluster of $>40$ events with a leukemic phenotype was considered as quantitative residual disease. If no cluster was detected, the result was termed "not detectable" at a given sensitivity level.

Molecular screening and MRD analysis by RQ-PCR was performed in accordance with EuroMRD guidelines using IG/TR gene rearrangements as targets and consensus primers[5]. DNA was extracted from mononuclear BM cells following Ficoll density centrifugation of the BM aspirate using the QIAamp MiniKit according to the manufacturers protocol.

\section{WGS, variant calling and target identification}

1,1 $\mu \mathrm{g}$ of gDNA from diagnostic BM samples was sequenced using a PCR-free, paired-end WGS protocol with a 30X coverage on an Illumina HiSeqX platform at Clinical Genomics, SciLifeLab, Stockholm, Sweden [20]. The Human GRCh37 (hg19) RefSeq [21] was used for annotation. SVs were identified using MIP [22] and visualised in the SCOUT interface [23]. MIP performs SV detection using CNVnator V0.3.2 [24] with $1 \mathrm{~kb}$ bins, Delly [25] TIDDIT V2.0.0 [26] as well as Manta [27]. Filtering was performed using a list of recurrent aberrations in ALL (Table 2 supplementary material). If no such aberration was detected in a sample, the CNV data from routine aCGH were scrutinized for events likely to result in novel leukemia- 
specific junction sequences. Candidate regions were then manually inspected in Integrative Genomics Viewer $[28,29]$. Whenever possible the junction sites created by SVs involving genes known to be recurrently involved in ALL were chosen as potential targets.

\section{ddPCR assay design}

Custom ddPCR assays covering SV breakpoint sequences were designed according to the Rare Mutation Detection Best Practices Guidelines (BioRad, Hercules, CA, USA) using Primer3Plus software [30]. When junctions were located in regions deemed unsuitable for primer design, manual design was attempted. InSilico PCR and Human BLAT Search [31] were executed to ensure the specificity of the assays. Primers and probes were purchased from Integrated DNA Technologies ${ }^{T M}$ (IDT, Coralville, lowa, USA). Amplicon sizes were $<100 \mathrm{bp}$. All target probes were labelled with 5' 6-FAM ${ }^{\mathrm{Tm}}$ fluorophores and $3^{\prime}$ lowa Black ${ }^{\circ} \mathrm{FQ}$ quenchers. Sequence details are available upon request. Off the shelf assays for reference genes $(A B C C 9$, or $A L B$ ) labelled with 5' HEX fluorophores and 3' BHQ-1 quenchers were purchased from BioRad. Assays were prepared with a final primer/probe concentration of $900 \mathrm{nM} / 250 \mathrm{nM}$ respectively and ddPCR reactions were run on the QX200 AutoDG Droplet Digital PCR System/QX200 Droplet Reader (BioRad, CA, USA) according to the manufacturer's instructions. All reactions contained a reference gene assay ( $A L B$ or $A B C C 9$ ) to control for DNA concentration and amplifiability.

\section{Assay optimization and performance testing}

Optimal annealing temperature was determined through a gradient-ddPCR on single and multiplexed assay reactions using diagnostic BM gDNA as template. A no template control (NTC, nuclease free water) and a negative control ( $\mathrm{gNC}$ ) was included in each run. Specificity and sensitivity was assessed by running $500 \mathrm{ng}$ from serial 10-fold dilutions of diagnostic BM gDNA from each patient in gNC (ranging from $10^{-1}$ to $\left.10^{-6}\right)$. All assays were individually tested for limit of detection (LoD) and limit of quantifiability (LoQ). Ultimately, minimal DNA input ddPCR reactions were performed in a multiplexed manner with $1 \mathrm{ng}$ of gDNA from $10^{-1}$ and $10^{-2}$ from BM gDNA dilutions (containing $100 \mathrm{pg}$ and $10 \mathrm{pg}$ of $\mathrm{PC}$, respectively).

\section{Quantification of targets in patient samples}

All DNA samples were loaded in triplicate ddPCR reactions. $11 \mu \mathrm{L}$ of cfDNA eluate or $500 \mathrm{ng}$ of gDNA from BM was loaded per well. Triplicates of controls were run on all plates (NTC, gNC and PC gDNA) with the addition of 9-12 wells of plasma NC for cfDNA analysis.

Output data were analyzed using the QuantaSoftPro/QX Manager Software (BioRad, Hercules, CA USA) and results were manually reviewed. Thresholds for empty (negative), target positive/reference negative, target negative/reference positive and double positive droplets were manually set based on the control samples included in each run according to the provider's instructions. For a sample to be considered target positive, three or more droplets with FAM-fluorescence were required. 1-2 positive droplets are referred to as trace amounts. 
The average copy number (CN) of target and reference molecules (FAM and HEX signals, respectively) per $\mathrm{ml}$ of liquid biopsy (plasma or CSF) was calculated by dividing the sum of all signals from the three replicate ddPCR reactions by initial liquid biopsy volume. The ratio of target molecules to reference molecules in cfDNA and gDNA (plasma and BM, respectively) was calculated after correction for the number of targets for each patient. In cases where BM samples from diagnosis were not available for ddPCR analysis (patients 1, 4 and 5), a theoretical ratio was extrapolated from the dilution series data.

\section{Results}

\section{WGS data enable target identification}

Our aim was to identify up to three targets per patient from the WGS data using the following criteria: i) events supported by reads that indicated their presence in a majority of leukemic blasts ii) unique sequences generated by SVs in the leukemic blasts, iii) recurrent stratifying genetic events alternatively putative driving events described as recurrent in the literature.

FISH results indicated a split signal pattern for TCF3 (19p13.3) in 53\% of BM cells for patient 1. The WGS data supported the presence of a reciprocal translocation between chromosomes 12 and 19, $t(12 ; 19)$ (p13;p13) and non-templated insertions at the junctions in both derivative chromosomes which were selected as targets (Fig. 2a). The rearrangement created a TCF3-ZNF384 fusion; reported as recurrent in ALL[32]. The sequence junctions were confirmed with Sanger sequencing (Fig. 2b). WGS data also revealed a deletion upstream of the KRAS gene on chromosome 12 (p12.1), including an eight bp nontemplated insertion at the junction site (Fig. 2c).

WGS results for patient 2 indicated intrachromosomal events on chromosome 9, resulting in deletions affecting both chromosome homologs. Polynucleotide stretches were found at the breakpoint junctions for both deletions. Nonetheless assays were attempted but failed to amplify specific PCR products. The WGS data detected two additional deletions, both affecting transcription factors essential for lymphocyte development and recurrent events in ALL; IKZF1 on chromosome 7 (p12.2) [33] and PAX5 on chromosome 9 (p13.2)[34] (Figure S1a-b supplementary material). The junction sequences resulting from these two deletions were selected as targets.

Routine analysis for patient $\mathbf{3}$ had revealed a high hyperdiploid clone with 54 chromosomes and several copy number alterations (CNAs) in 70\% of the BM cells. These CNAs including an extra derivative chromosome 8 were supported by the WGS data as well. However, inspection in IGV showed that most of these events had occurred in highly repetitive regions (Figure S2a supplementary material), and thus were inadequate as targets. Only one suitable SV junction resulting from an unbalanced rearrangement between chromosomes 2 and 8 was detected and was selected as the only target for patient 3 . In order to maximize the probability of detecting this target, a double probe assay was designed in which two (nonoverlapping) probes were located over the junction, one on the plus and one on the minus strand (Figure S2b supplementary material). 
For patient 4, the callers for SVs identified several aberrations affecting chromosome arms 4p, 9p, 20p and $21 \mathrm{q}$ in the WGS data. These events had also been detected by the routine methods and were all retained in both relapses. Out of these, three junction sequences were selected following inspection in IGV (Figure S3a-c supplementary material). The first target was the junction created by a deletion on chromosome arm 9p, encompassing $C D K N 2 A / B$. The WGS data indicated a pericentric inversion of chromosome 4 , with breakpoints in $4 p 14$ and $4 q 13.3$, resulting in a deletion together with a six base pair non-templated insertion at the junction on $4 \mathrm{p}$. This junction sequence was the second target selected. The third target, a deletion in chromosome 20 (p12.1p11.23) was selected since the WGS data showed an $11 \mathrm{bp}$ non-templated insertion at the junction site.

Routine cytogenetic analysis had identified a reciprocal translocation $\mathrm{t}(5 ; 7)(\mathrm{q} 35 ; \mathrm{q} 21)$ supporting a rearrangement affecting TLX3 and CDK6 recurrent in T-ALL [35] in patient 5. The WGS data also supported this translocation, however, as the resulting junctions were surrounded by highly repetitive sequences on $5 q$ and $7 q$ the assay design was rendered unfeasible. The WGS data also identified several SVs that resulted in deletions also detected by aCGH. The majority of these, with the exception of the homozygous deletion of the CDKN2A/B genes on 9p21.3, are not recurrent in ALL. Our final designs targeted SV junctions resulting from the deletion on $9 p$ as well as deletions affecting $8 p$ and $16 q$. (Figure S4a-c supplementary material)

For patient 6, CBA and aCGH had identified several structural events on chromosome 6 including an interstitial deletion (q14.1q15) previously described for driving T-cell leukemia progression[36], together with the recurrent deletion on chromosome 9p21.3 (including CDKN2A/CDKN2B). WGS data confirmed these events and revealed a STIL-TAL 1 fusion, a known recurrent event in T-ALL[37]. The junction sequences created by these rearrangements were chosen as targets (Figure S5a-c supplementary material).

\section{WGS based ddPCR assays detect targets down to a $10^{-6}$ dilution}

In total $18 \mathrm{ddPCR}$ assays were designed of which three failed, due to either lack of specificity or complex target regions with repetitive sequences. The 15 successful designs were further tested using serial 10fold dilutions of diagnostic BM gDNA in normal control gDNA from blood donors. No unspecific amplification was detected in DNA isolated from lymphocytes from normal healthy donors except for one target (PAX5 in patient 2), we thus adjusted the LoD to $10^{-5}$ for this target. The assays were evaluated for linearity and sensitivity to determine the range where the targets were quantifiable or detectable using $500 \mathrm{ng}$ input DNA. Linearity was achieved for all assays down to a $10^{-4}$ dilution, and eight out of 15 assays were linear down to a $10^{-5}$ dilution (representative example shown in Fig. 3 ). Thus, for four out of six patients LoQ reached $10^{-5}$, and in the remaining two patients the LoQ was $10^{-4}$. Seven of the targets were detectable one order of magnitude beyond the LoQ and all patients had at least one target that could be detected beyond the LoQ. The summary of LoDs and LoQs are shown in Table 2. 


\section{Assay performance at low gDNA input levels}

The assays were further tested in a multiplexed manner (1-3 targets/patient) in reactions containing low DNA input levels, i.e., $1 \mathrm{ng}$ DNA input per well (gDNA from $10^{-1}$ and $10^{-2}$ dilutions of PC in gNC). All tests were run in three replicate reactions. All targets were readily detectable at a $10^{-1}$ dilution containing 100 pg PC in 900 pg gNC. At the $10^{-2}$ dilution (10 pg PC gDNA in $990 \mathrm{pg} \mathrm{gNC}$ ) targets for half of the patients were still detectable while only trace signals ( $<3$ target positive droplets in three replicate wells) were detected for the remaining patients (Table 3). Moreover, the calculated $\mathrm{CN}$ were consistently below the theoretical CN calculated assuming no pipetting losses and 100\% PCR efficiency. We concluded that the assays could accurately quantify the targets also when DNA input was minimal (Table 3 supplementary material).

\section{cfDNA yield assessed by ddPCR}

The cfDNA level in the plasma samples was calculated from the reference gene copy number (CN) in the cfDNA eluate. After correcting for initial plasma volume and cfDNA eluate input volume, this was expressed as reference gene $\mathrm{CN} / \mathrm{ml}$ of plasma. Initially, some ddPCR reactions with cfDNA from plasma at diagnosis or relapse failed. High background fluorescence suggested that these reactions were oversaturated with cfDNA, as could be confirmed by titration of plasma volumes for cfDNA extraction, as well as cfDNA input volumes for ddPCR, until accurate cluster separation was achieved (Figure S6 supplementary material). This procedure was not required for follow-up plasma or CSF samples, which could be processed in their full volume.

cfDNA was recovered from all plasma samples collected $(n=53)$ but the yields varied greatly between sampling timepoints and patients. The levels in pre-treatment plasma samples differed 260 -fold between cases $\left(2.2 \times 10^{4}\right.$ to $5.8 \times 10^{6}$ reference gene $\left.\mathrm{CN} / \mathrm{ml}\right)$ and roughly paralleled the white blood cell count (WBCC). No plasma sample at diagnosis was available for patient 5 (T-ALL) but the highest cfDNA level was seen in patient 6 (also T-ALL) with $5.8 \times 10^{6}$ reference gene $\mathrm{CN} / \mathrm{ml}$. Overall, cfDNA levels in plasma decreased during therapy, however, the degree and timepoint varied between patients. In patients with higher $\mathrm{CN}$ in pre-treatment plasma $\left(>1 \times 10^{6} / \mathrm{ml}\right)$, who also had the highest WBCC at diagnosis, this decrease was more pronounced during induction. By the end of the first block of consolidation therapy (CB1), cfDNA levels had dropped to levels between $1.4 \times 10^{3}$ and $6.9 \times 10^{3}$ reference gene $\mathrm{CN} / \mathrm{ml}$, corresponding to an approx. 0.5 to $3 \log _{10}$ change (in patients 1 and 6, respectively) (Fig. 4).

Patients 1, 2 and 6 showed the largest discrepancies between the kinetics of BM MRD and plasma cfDNA. These patients had transient increases in total cfDNA plasma levels during follow-up due to severe complications. For patient 1 the increase coincided with severe treatment-related pancreatitis. The increase in patient 2 was seen after sepsis and renal insufficiency. Patient 6 showed an increase concomitant with severe treatment complications in the form of sepsis, coagulation system disorder and renal dysfunction. Taken together, these results indicate that the total cfDNA in plasma does not only reflect the leukemic burden but is influenced by several other pathophysiological processes and therefore 
a less suitable MRD marker in ALL. cfDNA was recovered from $96 \%$ of all CSF samples collected (51/53) and the amount reflected the quantity recovered from plasma for the BCP-ALL samples. In pre-treatment samples $(n=6)$ cfDNA levels ranged between 6 and 164 reference gene $\mathrm{CN} / \mathrm{ml}$. The two follow-up samples which gave no cfDNA yield were the smallest volumes in the series (150 and $300 \mu \mathrm{L}$, respectively). By CB1, cfDNA levels had dropped to minimal levels (1-5 reference gene $\mathrm{CN} / \mathrm{ml})$ in all patients.

\section{MRD level in BM samples by SV ddPCR assays}

Multiplexed ddPCR assays were used to quantify MRD in the same BM samples that had been used in routine diagnostics. Table 3 shows the MRD results by the experimental approaches and routine methods for comparison at the main timepoints used to stratify patients, end of induction (Eol) and end of consolidation block 1 (CB1). Unfortunately, DNA follow-up samples were not available for ddPCR for patients 5 and 6 . At Eol all available BM samples that were MRD positive by routine methods (FCM and/or IG/TR RQ-PCR) were also positive by SV ddPCR. The level of MRD was in a comparable range between methods for samples with detectable residual disease, although our approach consistently detected higher values for the four patients with BCP-ALL. Patient number 3 , that had undetectable MRD by FCM (RQ-PCR data not available), was positive by ddPCR. Also, patient 4 who later suffered a relapse despite being transferred to the IR group, showed a 3-fold higher MRD level with ddPCR than with FCM.

At CB1 the results were also concordant, although again for three out of five patients without detectable MRD by FCM, ddPCR showed low levels within the quantifiable range. Interestingly, patient 4 still had detectable MRD by ddPCR $(0,03 \%)$ at this timepoint, while no LAIP was detected by FCM in the same sample. Taken together, the results suggest that our approach to design molecular assays, together with ddPCR quantification, may result in a more sensitive MRD extending the range of quantifiability of leukemic targets.

We also investigated the leukemic burden measured in plasma ctDNA with the ddPCR assays at the same timepoints. The results follow the same pattern and all but one of the samples that scored positive in BM DNA were also positive in plasma cfDNA (Table 3). Interestingly, for patient 1 the ctDNA results indicated higher level of residual disease than did the BM ddPCR results.

\section{Kinetics of leukemic targets in plasma by ddPCR}

The ratio between multiplexed leukemic target $\mathrm{CN}$ and reference gene $\mathrm{CN}$ was calculated as described earlier. These ratios were plotted together with MRD information from routine evaluation during the first months following diagnosis (Fig. 5). Overall, the level of targets decreased in ctDNA over time during induction and consolidation following the MRD level detected in BM. We found that patients 1 and 3 had higher ratios in plasma than in BM, although the difference was more pronounced by d15 than at the Eol timepoint. In contrast, patients 4, 5 and 6 cleared the targets faster in plasma than in the BM (FCM) at all timepoints investigated. Only for patient 2, the level of target measured in plasma shifted from below the MRD in BM by d15 to above by Eol and again below BM MRD at the later timepoint. 
Patient 4 suffered two relapses, and we thus investigated the kinetics of ctDNA over the entire course of disease. (Fig. 5 panel d). As seen for the other patients the disease burden quickly decreased during induction, reaching no detectable level by CB1. During subsequent follow up only the samples taken d176 and d372 showed trace amounts of target. Interestingly, by d428 the plasma ratio increased markedly to $31 \%$, two months prior to overt relapse (d489) which was diagnosed when the patient was admitted to ER/hospital due to pain. By then, both plasma and BM showed high ratios of leukemic targets $(61 \%$ and $97 \%$, respectively). HR relapse treatment was initiated and over the next month the plasma ratio dropped to $0.5 \%$ (d518). Interestingly, the ratio in BM dropped to a minimum three weeks later, (d539; $2.5 \%$ ), while plasma ratio was again increasing in the sample taken at this timepoint. Stem cell transplantation (SCT) was performed (d620) but the patient was later readmitted (d689) due to suspect post-transplantation relapse. By then, plasma ratio was $44 \%$ and remained around this level until the last sampling timepoint, one month before the patient passed away.

\section{Detection of leukemia targets in cfDNA from CSF}

None of the six patients had CNS involvement based on morphological examination of the CSF, however FCM had detected cells with LAIP in patient 2. cfDNA was successfully extracted from all diagnostic CSF samples and leukemic targets were detected in half of these, including patient 2 (Table 4). Target-positive CSF samples also had more copies of the reference gene than negative samples (34-164 and 6-16 reference gene $\mathrm{CN} / \mathrm{ml}$, respectively), indicating that more cells had contributed cfDNA in those samples.

Although there was a correlation between high pre-treatment levels of leukemic targets in plasma and CSF positivity in some patients, this was not consistent. For example, patient 3 had few copies of the reference gene and no leukemic targets in the CSF despite having high levels of both in plasma at diagnosis. In patient 4, who was positive for leukemic targets in the CSF at diagnosis, only trace amounts were found in later samples with no correlation to the level in the paired plasma samples. Taken together, these results suggest that leukemic targets in the CSF do not solely reflect leakage from the plasma but possibly signal the presence of malignant cells inside the blood brain barrier.

\section{Discussion}

High-throughput technologies, in particular WGS, have shown huge potential to identify the genomic aberrations that define genetic subgroups in ALL [9] and are increasingly being introduced in the diagnostic setting for hematological malignancies [10-15]. The present study was designed to explore the possibility to extend the diagnostic utility of WGS beyond genetic characterization. Since MRD assessment is the most important parameter in risk adapted treatment protocols, we investigated the feasibility to use WGS to identify genomic structural rearrangements that involve breakage and reunion of the genome and use the novel sequences generated at the SV junctions as leukemia-specific unique markers. We hypothesized that genes recurrently rearranged in ALL are likely to be important for leukemic 
evolution and/or leukemia maintenance and would thus be preserved during the course of the disease and therefore constitute suitable targets to monitor treatment response.

The patients in the study were selected to challenge WGS; none of the BCP-ALL harbored the recurrent rearrangements that result in fusion genes mandatory to investigate in the NOPHO 2008 treatment protocol. Nonetheless, WGS identified suitable targets in all BCP-ALL patients and in the two T-ALL patients included. WGS enabled the detailed characterization of the breakpoints and directly provided the leukemia-specific junction sequences. Some of the chromosomal rearrangements first selected had occurred at genomic sites with highly repetitive sequences and were unsuitable as targets. This was particularly evident in patient 3, a BCP-ALL with high hyperdiploidy, where all structural events but one, had occurred in such regions. This phenomenon is a known feature of the human genome: SV are enriched in regions that contain repetitive regions[38]. Patient 3 was the only patient where the one suitable clonal marker was not in a genomic region reported to be involved in the pathogenesis of ALL. In order to boost the sensitivity, a single assay with double probes was designed and resulted in a LoQ and LoD comparable with the other patients in the study.

The current "gold standard" for molecular monitoring of residual disease in ALL is to measure clonal IG/TR rearrangements with allele-specific quantitative PCR, a demanding method that has been extensively standardized by the EuroMRD consortium[5]. In addition, this method requires a substantial amount of BM DNA and was the reason why BM DNA samples were not available from all time points in the patients with T-ALL. We used the criteria recommended by EuroMRD to test the performance of the SV ddPCR assays on BM. Four out of six patients LoQ reached $10^{-5}$, and in the remaining two patients the LoQ was $10^{-4}$. All patients had at least one assay able to detect one leukemic cell in 100,000 normal cells and the limiting factor for the sensitivity was the input DNA. Thus, the assays were at least as good as and potentially superior to IG/TR-PCR in regards of specificity, sensitivity and range of quantifiability and had no background amplification from the germline. They also showed good concordance with routine MRD methods, although for a few samples ddPCR indicated higher MRD values. This was particularly evident for patient 4, where ddPCR showed MRD above the routine methods at both Eol and CB1 timepoints, despite the fact that this patient's assays had the lowest LoD/LoQ.

All targets selected were present in a high proportion of leukemic blasts, and when possible recurrent genetic aberrations, and thus likely represented truncal events. The use of several targets per patient minimizes the risk that a genetic event will be lost during clonal evolution. In order to maximize the accuracy and sensitivity when few copies of the targets are present the assays were successfully multiplexed for all patients except in patient 3 where the high hyperdiploid clone only rendered one target. In all, ddPCR assays based on SVs were highly specific which enabled precise MRD quantification with very little optimization and no need for standard curves. Hence, this WGS-based approach enabled simultaneous genetic characterization of the leukemic blasts with regards to recurrent genetic markers and identification of specific molecular targets to monitor therapy response. 
The successful clinical implementation of WGS for the diagnostics of germline conditions has paved the way for introduction of WGS in the diagnostic setting of malignancies[39, 40]. In pioneering studies, WGS, often combined with WTS, has been evaluated as a diagnostic tool in acute leukemia with promising results. Compared with standard of care multi-testing, WGS/WTS performed equally well or better in identifying clinically relevant genetic aberrations in acute leukemia patients and changed the risk classification in a proportion of cases $[15,41]$. Within Genomic Medicine Sweden, a national study evaluating WGS and WTS in acute leukemia diagnostics is ongoing, with the ultimate aim to replace standard of care methods [42] (Berglund et al., unpublished results). With decreasing sequencing costs, these type of studies will hopefully provide the required impetus to gradually implement these powerful techniques in the diagnostic setting.

A test strategy for personalized treatment protocols based on WGS is often considered premature for clinical routine due to the still relatively time-consuming methods. However, the strategy outlined in Fig. 1 is suitable for MRD testing since there is enough time to perform and analyze the WGS data and to design and evaluate different ddPCR targets before MRD monitoring is of clinical relevance.

The results also show that with our approach, MRD can be successfully measured in cfDNA from a blood sample with a sensitivity comparable to that in BM. To our knowledge, this is the first time that MRD has been monitored in cfDNA using patient-specific targets determined from WGS on BM in ALL. Using targeted ddPCR, we observed extremely high levels of ctDNA in plasma samples at diagnosis and the kinetics showed a progressive decline during therapy. However, total cfDNA is unspecific as a MRD marker since it can be affected by other medical conditions as seen in three of our patients. On the other hand, sensitive detection and quantification of the patient-specific SV targets were possible. The selected targets decreased in plasma during the induction and initial consolidation, reflecting the leukemic burden.

The plasma markers rose from being undetectable in plasma to very high levels 2 months before overt recurrence in the only patient in this series that suffered a relapse. This result is in line with the results from other studies that suggest that ctDNA may be a sensitive tool to monitor therapy response in hematological malignancies[43,44] and that ctDNA not only reflects the circulating malignant cells but rather represents the entire disease burden [45]. Although residual leukemic cells can be detected in peripheral blood, they do not consistently represent the residual disease in the bone marrow and reliable MRD monitoring today calls for bone marrow sampling[46]. However, monitoring leukemia burden through plasma ctDNA in ALL could potentially be more representative than BM aspiration and has the additional advantage of being less invasive than BM sampling which, in children, is performed under general anesthesia.

Of note, the targeted SVs could be detected in CSF from half of the patients and $1 \mathrm{ml}$ CSF was sufficient. Our results suggest that the presence of ctDNA in CSF is not solely the consequence of leakage from the plasma, but rather correlates to the presence of malignant cells in the CSF. There were no traumatic punctures in the study, and as the CSF was collected in STRECK tubes containing cell stabilizing agents any leukemic cells from minimal puncture bleedings are not likely to have caused false positives. The 
current methods for detection of CNS involvement based on morphology miss a large number of patients with leukemic cells in the CNS and therefore all children are treated with intrathecal prophylaxis[7, 8]. Studies on FCM to improve CNS diagnostics are ongoing and the potential contribution of ctDNA analysis on CSF also needs to be evaluated in a larger cohort.

A potential limitation of our approach is that SVs can occur in genomic regions harboring repetitive sequences as was the case with patient 3 with a had high hyperdiploid karyotype. Despite detection of multiple SVs in the WGS data only one suitable ddPCR target was identified. Thus, in patients where the class defining aberration is an aneuploidy it may be difficult to find several suitable targets. A limitation for all molecular methods is the turn-around time; assays need to be available by end of induction to be useful for clinical decision-making. As WGS provides data for both genetic classification and SV target identification in one seamless workflow, and SV ddPCR requires minimal optimization, this approach is time efficient.

\section{Conclusions}

WGS enabled development of sensitive patient-specific ddPCR assays using the unique sequences generated by the chromosomal rearrangements associated with leukemia pathogenesis. The study shows that WGS not only detects the recurrent aberrations mandatory in treatment protocols but also directly identifies leukemia-specific molecular markers and that custom ddPCR assays show promising results for MRD monitoring. Moreover, the results on ctDNA suggest that monitoring the leukemia markers in plasma using ddPCR may contribute valuable information to the individualized follow-up of ALL patients in a minimally invasive manner. Further studies to systematically investigate the biology of ctDNA in ALL and its potential as a MRD marker in plasma and CSF in larger patient cohorts within current treatment protocols are warranted.

\section{Abbreviations}

ALL acute lymphoblastic leukemia

aCGH comparative genome hybridization array

AML acute myeloid leukemia

BM bone marrow

CB1 consolidation block 1

CBA chromosome band analysis

cfDNA cell free DNA

CNA copy number alteration 
CNS central nervous system

CSF cerebrospinal fluid

cfDNA cell free DNA

ctDNA cell free tumor DNA

ddPCR digital droplet polymerase chain reaction

Eol End of induction

FCM flow cytometry

FISH fluorescence in situ hybridization

gNC normal control genomic DNA

HR high risk

IG immunoglobulin

IGV Integrated Genome Viewer

IR intermediate risk

LAIP leukemia associated immunophenotype

MRD measurable residual disease

NIPT non-invasive prenatal testing

NOPHO Nordic Organization Pediatric Hematology and Oncology

NTC no template control

PB peripheral blood

PC positive control

RQ-PCR real-time quantitative polymerase chain reaction

SR standard risk

SV structural variants

TR T-cell receptor 
WBCC white blood cell count

WGS whole genome sequencing

\section{Declarations}

\section{Ethics approval and consent to participate}

The study was approved by the Ethical Review Board at Stockholm County and written informed consent was obtained from the patients' guardians at the time of inclusion. The study complies with the principles of the Helsinki Declaration.

\section{Consent for publication}

Not applicable.

\section{Availability of data and materials}

The experimental results from the study are included in the manuscript or in the supplementary information. The raw datasets generated by WGS are not publicly available due patient's privacy but the analyzed datasets that support the findings are available from the corresponding author on reasonable request.

\section{Competing interests}

The authors declare that they have no competing interests.

\section{Funding}

We are thankful for the support from the Swedish Childhood Cancer Fund through the following grants: TJ2018-0054 and TJ2021-0125, KP2018-0009, TJ 2015-0047, PR 2017-0063, TJ2018-0012 Barncancerfonden. We would also like to thank Mary Béves forskningsfond and ALF/Region Stockholm: SLL20200306, SLL20180046.

\section{Contributions}

CA and FR performed all experiments, interpreted the data and wrote the manuscript. AHS and NM collected the study samples and clinical data. LS performed the morphological examination and FCM analysis. RR and MN contributed to study design and interpretation of results. ET and GB conceived the study, supervised the work and contributed to manuscript writing. All authors read and approved the final manuscript.

\section{Acknowledgements}


The authors would like to acknowledge the patients and their families, the staff at the Pediatric Oncology Unit as well as the Clinical Genetics Department at Karolinska University Hospital. We are also grateful to the staff at the Clinical Genomics Platform at Science for Life Laboratory (SciLife Lab), Stockholm, particularly to Valtteri Wirta and Jesper Eisfeldt, for expert assistance regarding WGS and bioinformatics.

\section{References}

1. Inaba, H. and C.G. Mullighan, Pediatric acute lymphoblastic leukemia. Haematologica, 2020. 105(11): p. 2524-2539.

2. Borowitz, M.J., et al., Prognostic significance of minimal residual disease in high risk B-ALL: a report from Children's Oncology Group study AALL0232. Blood, 2015. 126(8): p. 964-71.

3. Toft, N., et al., Results of NOPHO ALL2008 treatment for patients aged 1-45 years with acute lymphoblastic leukemia. Leukemia, 2018. 32(3): p. 606-615.

4. Theunissen, P., et al., Standardized flow cytometry for highly sensitive MRD measurements in B-cell acute lymphoblastic leukemia. Blood, 2017. 129(3): p. 347-357.

5. van der Velden, V.H., et al., Analysis of minimal residual disease by Ig/TCR gene rearrangements: guidelines for interpretation of real-time quantitative PCR data. Leukemia, 2007. 21(4): p. 604-11.

6. Kim, I.S., Minimal residual disease in acute lymphoblastic leukemia: technical aspects and implications for clinical interpretation. Blood Res, 2020. 55(S1): p. S19-s26.

7. Ranta, S., et al., Detection of central nervous system involvement in childhood acute lymphoblastic leukemia by cytomorphology and flow cytometry of the cerebrospinal fluid. Pediatr Blood Cancer, 2015. 62(6): p. 951-6.

8. Pui, C.H. and E. Thiel, Central nervous system disease in hematologic malignancies: historical perspective and practical applications. Semin Oncol, 2009. 36(4 Suppl 2): p. S2-s16.

9. Kimura, S. and C.G. Mullighan, Molecular markers in ALL: Clinical implications. Best Pract Res Clin Haematol, 2020. 33(3): p. 101193.

10. Smadbeck, J.B., et al., Copy number variant analysis using genome-wide mate-pair sequencing. Genes Chromosomes Cancer, 2018. 57(9): p. 459-470.

11. Kuo, F.C., et al., The relative utilities of genome-wide, gene panel, and individual gene sequencing in clinical practice. Blood, 2017. 130(4): p. 433-439.

12. Brüggemann, M. and M. Kotrova, Minimal residual disease in adult ALL: technical aspects and implications for correct clinical interpretation. Blood Adv, 2017. 1(25): p. 2456-2466.

13. Tran, T.H. and S.P. Hunger, The genomic landscape of pediatric acute lymphoblastic leukemia and precision medicine opportunities. Semin Cancer Biol, 2020.

14. Nordlund, J., et al., Refined detection and phasing of structural aberrations in pediatric acute lymphoblastic leukemia by linked-read whole-genome sequencing. Sci Rep, 2020. 10(1): p. 2512.

15. Duncavage, E.J., et al., Genome Sequencing as an Alternative to Cytogenetic Analysis in Myeloid Cancers. N Engl J Med, 2021. 384(10): p. 924-935. 
16. Schwarz, A.K., et al., Quantification of free total plasma DNA and minimal residual disease detection in the plasma of children with acute lymphoblastic leukemia. Ann Hematol, 2009. 88(9): p. 897-905.

17. Cheng, S.H., et al., Minimal residual disease-based risk stratification in Chinese childhood acute lymphoblastic leukemia by flow cytometry and plasma DNA quantitative polymerase chain reaction. PLoS One, 2013. 8(7): p. e69467.

18. Oncology, N.S.o.P.H.a., NOPHO ALL-2008 final protocol version 1b-3. 2008.

19. ALLTogether study - A Treatment Protocol for Participants 1-45 Years With Acute Lymphoblastic Leukaemia.

20. Clinical Genomics, Stockholm, Sweden. Available from: https://www.scilifelab.se/facilities/clinicalgenomics-stockholm/.

21. Genome Reference Consortium Human Build 37.

22. MIP - Mutation Identification Pipeline. Available from: https://mip-api.readthedocs.io/en/latest/.

23. Scout. Available from: https://github.com/Clinical-Genomics/scout.

24. Abyzov, A., et al., CNVnator: an approach to discover, genotype, and characterize typical and atypical CNVs from family and population genome sequencing. Genome Res, 2011. 21(6): p. 974-84.

25. Rausch, T., et al., DELLY: structural variant discovery by integrated paired-end and split-read analysis. Bioinformatics, 2012. 28(18): p. i333-i339.

26. Eisfeldt, J., et al., TIDDIT, an efficient and comprehensive structural variant caller for massive parallel sequencing data. F1000Res, 2017. 6: p. 664.

27. Chen, X., et al., Manta: rapid detection of structural variants and indels for germline and cancer sequencing applications. Bioinformatics, 2016. 32(8): p. 1220-2.

28. Robinson, J.T., et al., Integrative genomics viewer. Nat Biotechnol, 2011. 29(1): p. 24-6.

29. Thorvaldsdóttir, H., J.T. Robinson, and J.P. Mesirov, Integrative Genomics Viewer (IGV): highperformance genomics data visualization and exploration. Brief Bioinform, 2013. 14(2): p. 178-92.

30. Untergasser, A., et al., Primer3Plus, an enhanced web interface to Primer3. Nucleic Acids Res, 2007. 35(Web Server issue): p. W71-4.

31. Kent, W.J., BLAT-the BLAST-like alignment tool. Genome Res, 2002. 12(4): p. 656-64.

32. Hirabayashi, S., et al., ZNF384-related fusion genes define a subgroup of childhood B-cell precursor acute lymphoblastic leukemia with a characteristic immunotype. Haematologica, 2017. 102(1): p. 118-129.

33. Marke, R., F.N. van Leeuwen, and B. Scheijen, The many faces of IKZF1 in B-cell precursor acute lymphoblastic leukemia. Haematologica, 2018. 103(4): p. 565-574.

34. Medvedovic, J., et al., Pax5: a master regulator of B cell development and leukemogenesis. Adv Immunol, 2011. 111: p. 179-206.

35. Huret, J.L., et al., Atlas of genetics and cytogenetics in oncology and haematology in 2013. Nucleic Acids Res, 2013. 41(Database issue): p. D920-4. 
36. Gachet, S., et al., Deletion 6q Drives T-cell Leukemia Progression by Ribosome Modulation. Cancer Discov, 2018. 8(12): p. 1614-1631.

37. Janssen, J.W., et al., SIL-TAL 1 deletion in T-cell acute lymphoblastic leukemia. Leukemia, 1993. 7(8): p. 1204-10.

38. Audano, P.A., et al., Characterizing the Major Structural Variant Alleles of the Human Genome. Cell, 2019. 176(3): p. 663-675.e19.

39. Stranneheim, H., et al., Integration of whole genome sequencing into a healthcare setting: high diagnostic rates across multiple clinical entities in 3219 rare disease patients. Genome Med, 2021. 13(1): p. 40.

40. Rosenquist, R., et al., Clinical utility of whole-genome sequencing in precision oncology. Semin Cancer Biol, 2021.

41. Rusch, M., et al., Clinical cancer genomic profiling by three-platform sequencing of whole genome, whole exome and transcriptome. Nat Commun, 2018. 9(1): p. 3962.

42. Hellström Lindberg, E., et al., [Precision diagnostics and therapy in hematological malignancies]. Lakartidningen, 2021. 118.

43. Iriyama, C., et al., Using peripheral blood circulating DNAs to detect CpG global methylation status and genetic mutations in patients with myelodysplastic syndrome. Biochem Biophys Res Commun, 2012. 419(4): p. 662-9.

44. Zhong, L., et al., Monitoring immunoglobulin heavy chain and T-cell receptor gene rearrangement in cfDNA as minimal residual disease detection for patients with acute myeloid leukemia. Oncol Lett, 2018. 16(2): p. 2279-2288.

45. Yeh, P., et al., Circulating tumour DNA reflects treatment response and clonal evolution in chronic lymphocytic leukaemia. Nat Commun, 2017. 8: p. 14756.

46. Coustan-Smith, E., et al., Use of peripheral blood instead of bone marrow to monitor residual disease in children with acute lymphoblastic leukemia. Blood, 2002. 100(7): p. 2399-402.

\section{Tables}

Table 1 to 4 are available in the Supplemental Files section.

\section{Figures}


Figure 1

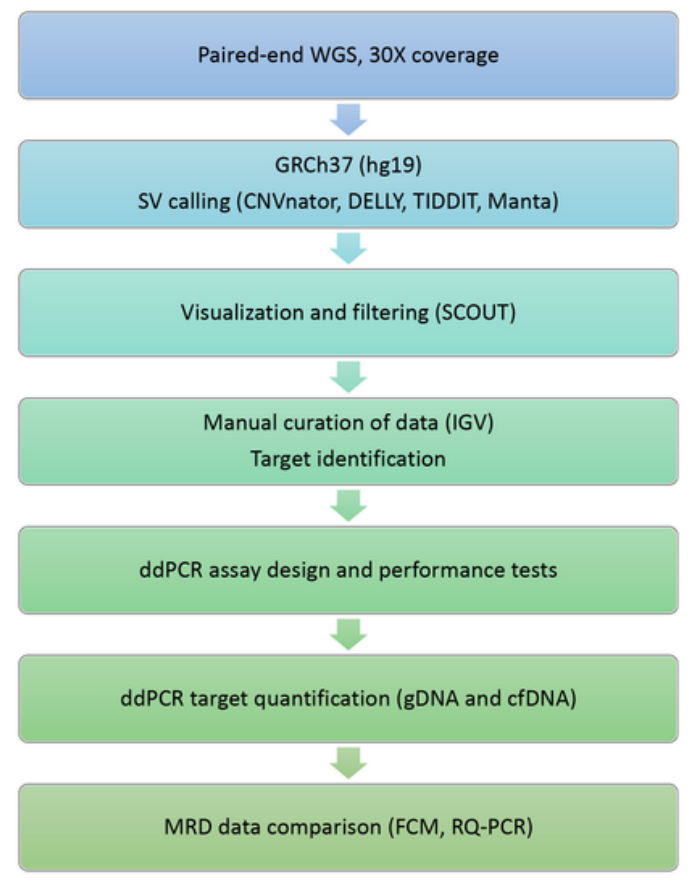

\section{Figure 1}

Flow chart of steps and techniques used for identification and quantification of leukemic targets.

WGS, whole genome sequencing; GRCh37, Genome Reference Consortium Human Build 37; SV, structural variant; IGV, The Integrative Genomics Viewer; ddPCR, droplet digital polymerase chain reaction; gDNA, genomic DNA; cfDNA, cell free DNA; MRD, measurable residual disease; FCM, flow cytometry; RQ-PCR, real-time quantitative polymerase chain reaction. 
Figure 2
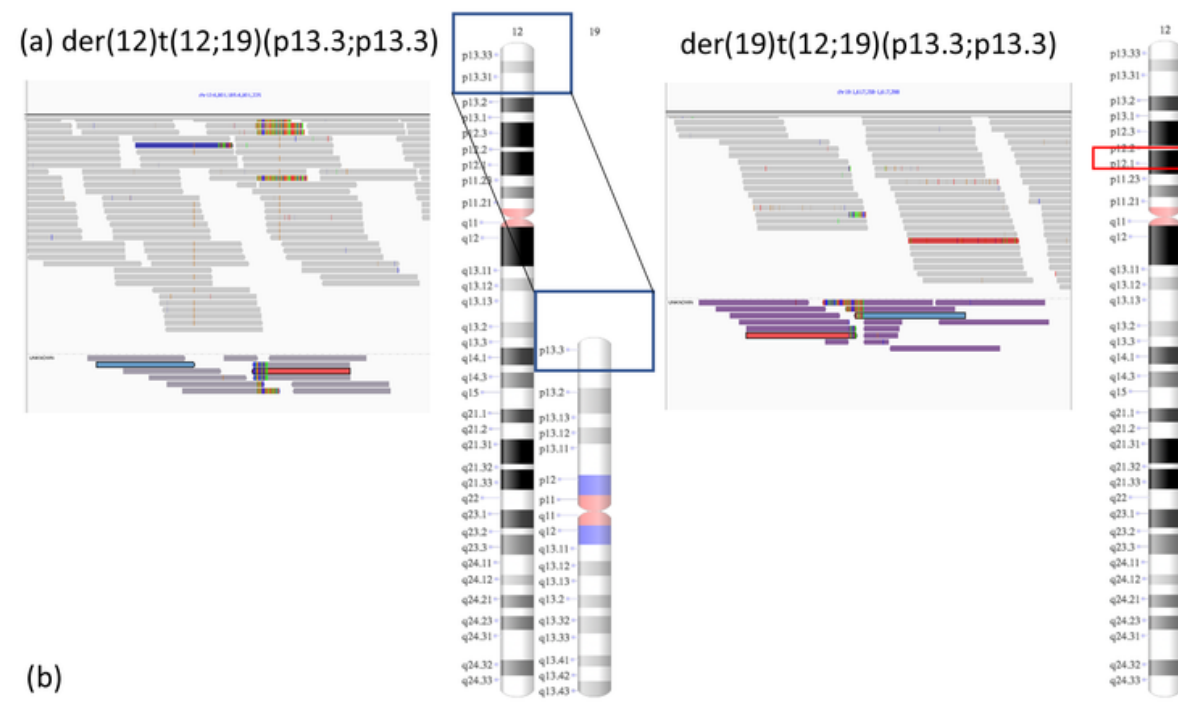

(c) del(12)(p12.1) upstream of KRAS

(b)

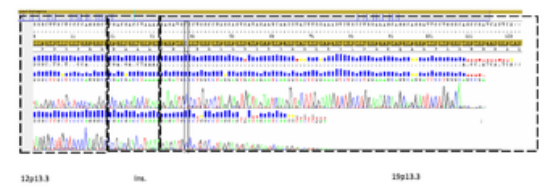

$$
\text { sol }
$$

sal

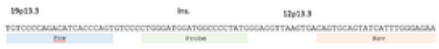

\section{Figure 2}

Target sequences selected for patient 1. (a) Screen shots from IGV showing the junction sequences on derivative chromosomes 12 and 19 and ideogram representing the regions involved in the reciprocal translocation. Discordant reads are marked in medium grey (ZNF384 locus) and purple (TCF3 locus).

(b) Sanger sequencing confirmed the sequence to be identical to the sequence derived from WGS data, including the non-templated insertion in both junctions (sequences within broken lines).

(c) Screen shots from IGV (discordant reads in red) and ideogram representing the deletion on 12p12.1 (upstream of KRAS). 
Figure 3a

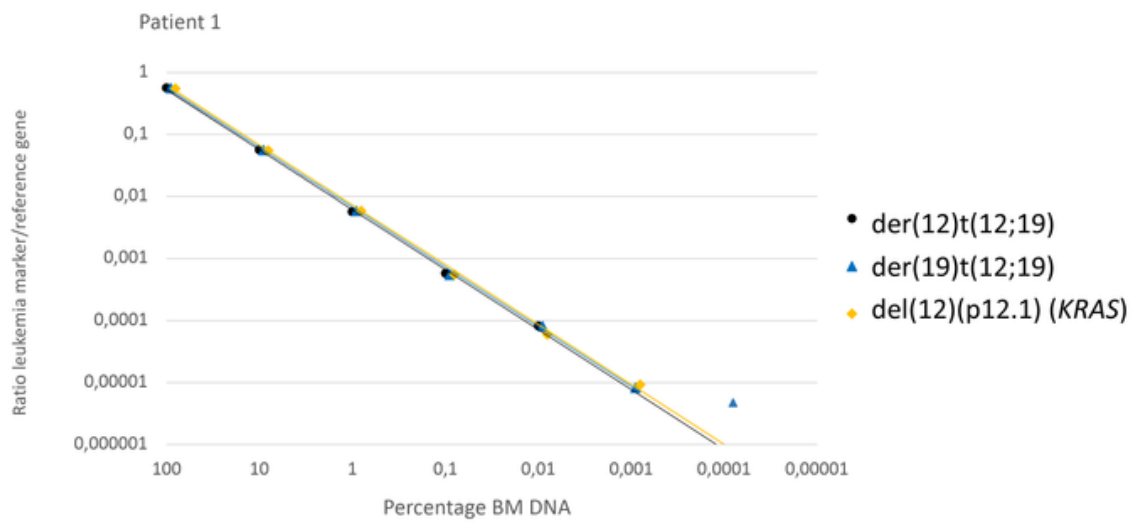

Figure $3 b$

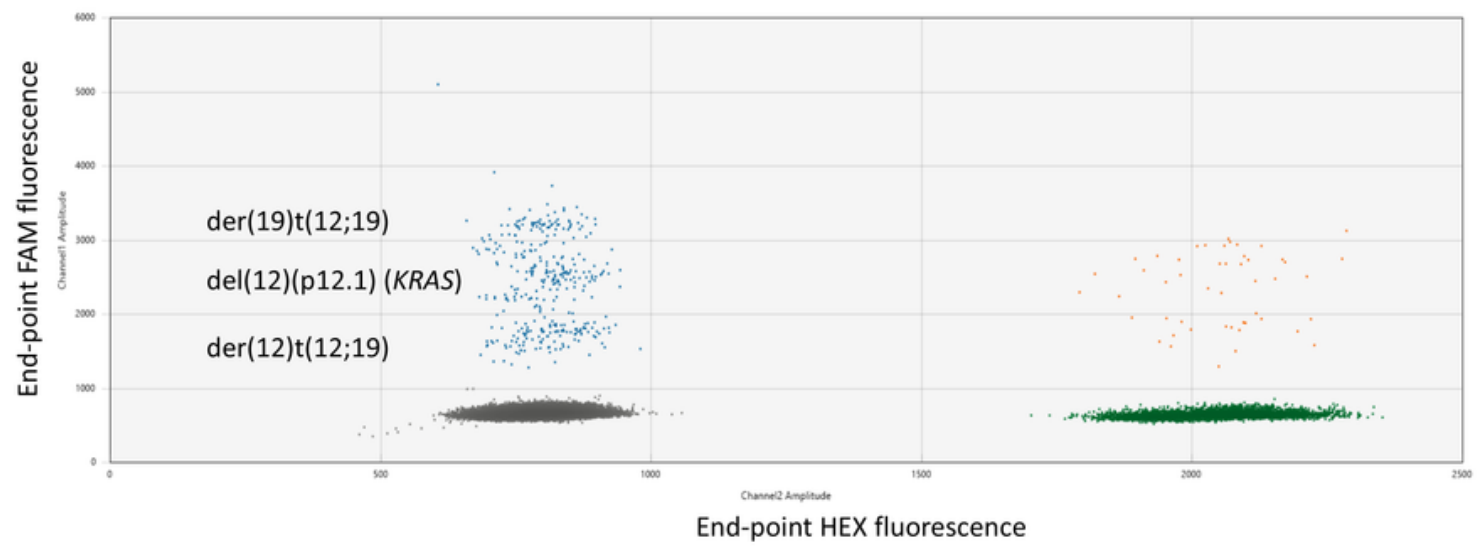

Figure 3

Dilution series and ddPCR fluorescence plot for leukemic targets in patient 1. (a) Sensitivity testing for the three targets selected for patient $1 ; \operatorname{der}(12), \operatorname{der}(19)$ and deletion $12 \mathrm{p}$. The ratios $\mathrm{CN}$ target to $\mathrm{CN}$ reference obtained from the ddPCR assays are plotted against the percentage of gDNA from the diagnostic BM for the different dilutions using 500 ng input gDNA. 
(b) 2D fluorescence amplitude plot generated by the QuantaSoft ${ }^{\mathrm{TM}}$ software showing the droplet clusters corresponding to the three different targets for patient 1 (der(12), der(19) and deletion 12p using $1 \mathrm{ng}$ of diagnostic BM gDNA in a background of $9 \mathrm{ng}$ gNC. Y-axis, end-point FAM-fluorescence from targets. Xaxis, end-point HEX-fluorescence from reference gene. Negative droplets (grey). Target positive-reference negative droplets (blue). Reference positive-target negative droplets (green). Double positive droplets (orange).

Figure 4

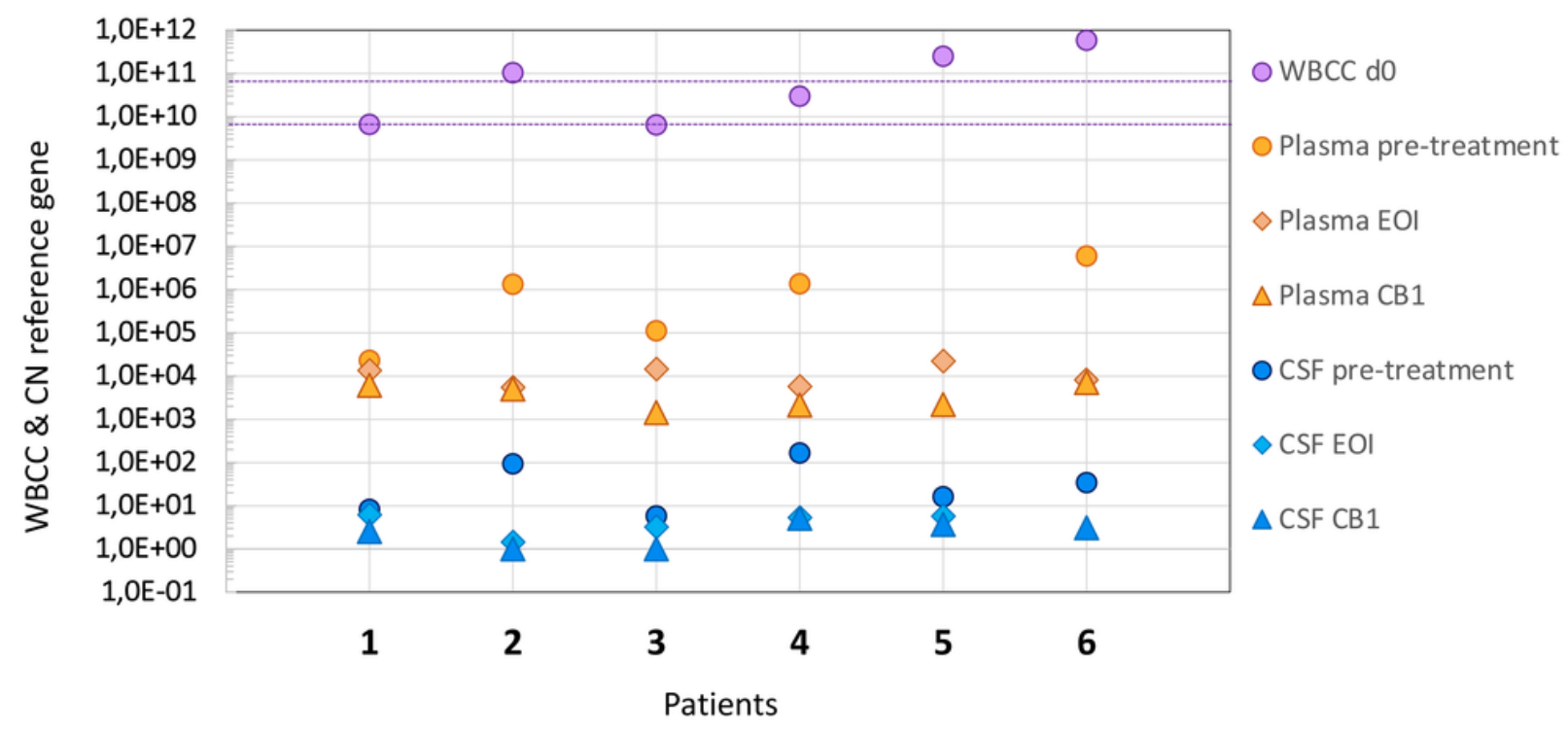

\section{Figure 4}

WBCC and cfDNA levels in plasma and CSF. cfDNA level expressed as CN of reference gene/ml in plasma (orange symbols) and in CSF (blue symbols) in pre-treatment samples (circles), at the end of induction (Eol) (diamonds) and at the end of first block of consolidation therapy (CB1) (triangles). Purple circles indicate the WBCC at presentation (cells/L). The reference range for WBCC is 5.0-15.0 $\times 10^{9} / \mathrm{L}$. 

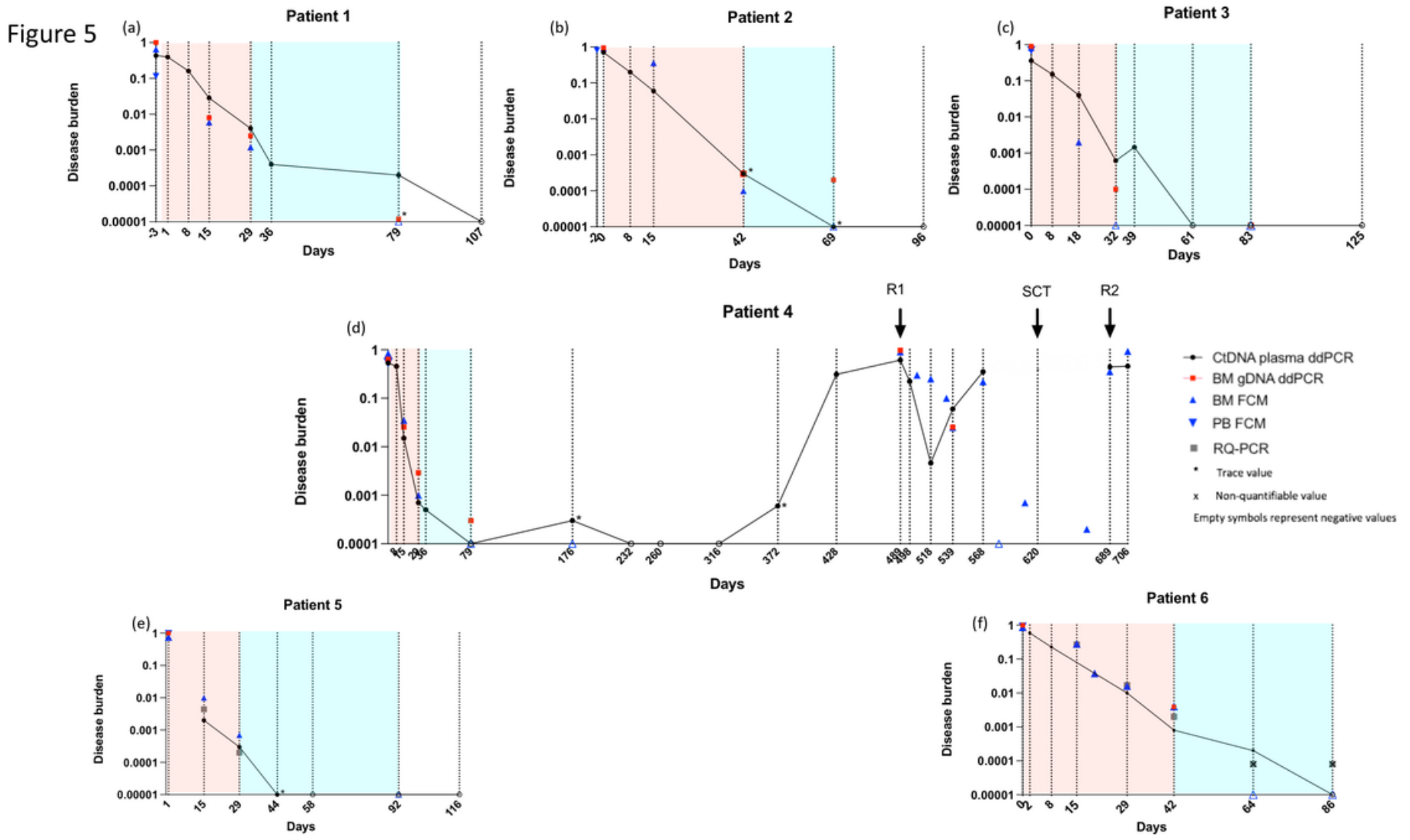

\section{Figure 5}

Kinetics of leukemic targets in plasma and BM by ddPCR and routine evaluation methods. Plasma ctDNA ratio by ddPCR (black circles). BM gDNA ratio by ddPCR (red squares). Asterisk denote trace amounts ( $<3$ positive droplets). Empty symbols denote negative values. Cells with LAIP by FCM in BM (blue triangles) and in PB (inverted blue triangles). In the panels from patients 5 and 6 (both T-ALL) qPCR data is shown (grey squares). Note that the lower level of detection for the qPCR assay is 0.0001 ; qPCR positive, nonquantifiable samples (black cross). 
Figure 6a

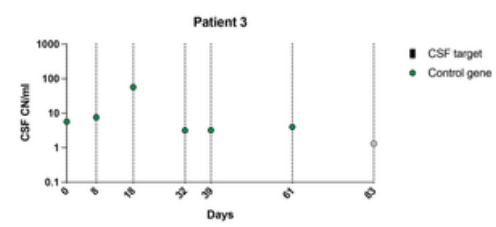

Figure 6b

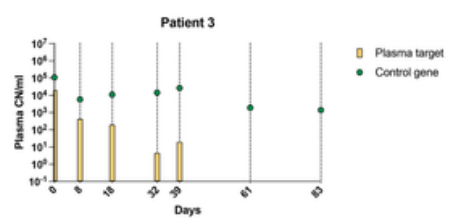

Figure $6 c$

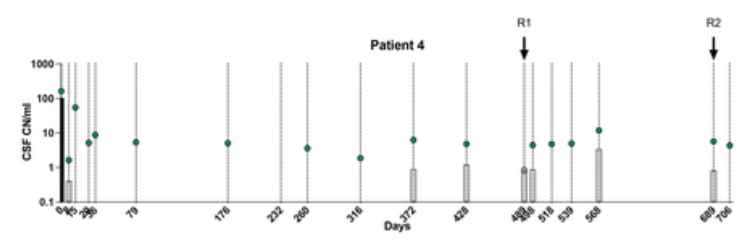

Figure 6d

1. Plasma al targess
- Control pone

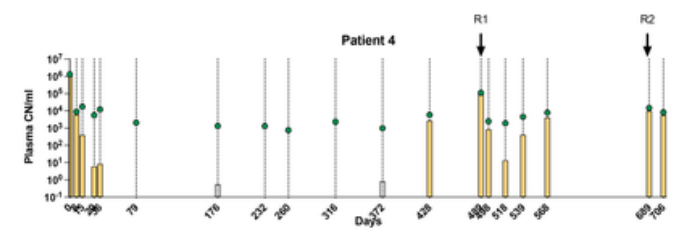

\section{Figure 6}

$\mathrm{CN} / \mathrm{ml}$ of leukemic target and reference gene molecules in CSF during treatment. (a) CSF and (b) plasma results in patient 3. (c) CSF and (d) plasma results in patient 4. Empty symbols denote trace values (1-2 positive droplets).

\section{Supplementary Files}

This is a list of supplementary files associated with this preprint. Click to download.

- Suppl.fig.15targets220208.pdf

- Table1patientcharacteristics220204.xlsx

- Table2assayperformance220204.xlsx

- Table3MRD220209.xlsx

- Table4CSFresults220204.xlsx 